\title{
EXTRACCIÓN ASISTIDA MEDIANTE LITOTRICIA EXTRACORPÓREA DE UN CATÉTER URETERAL CALCIFICADO OLVIDADO 2 AÑOS
}

\author{
F. MILLÁN RODRÍGUEZ, F. ROUSAUd BARÓN, J. MARTÍ MALET*, \\ F. IZGUIERDO LATORRE*, A. ROUSAUD BARÓN
}

Servicio de Urología. *Servicio de Radiología. Fundación Puigvert. Barcelona.

Actas Urol Esp. 27 (4): 301-304, 2003

\section{RESUMEN}

"EXTRACCIÓN ASISTIDA MEDIANTE LITOTRICIA EXTRACORPÓREA DE UN CATÉTER URETERAL CALCIFICADO OLVIDADO 2 AÑOS"

Se presenta el caso de un varón de 45 años que llevaba olvidado durante 2 años un catéter ureteral doble $\mathrm{J}$ izquierdo. El catéter presentaba ambos extremos calcificados no pudiéndose extraer mediante cistoscopia y pinzas. Se realizaron sesiones de litotricia extracorpórea en ambos extremos consiguiéndose la fragmentación de la litiasis y posteriormente pudo ser extraído en dos trozos mediante cistoscopia y ureteroscopia.

PALABRAS CLAVE: Catéter ureteral. Calcificación. Litotricia extracorpórea.

\section{ABSTRACT}

"EXTRACORPOREAL WAVE LITHOTRIPSY ASSISTED DRAWING OF A CALCIFIED URETERAL STENT LEFT OUT FOR TWO YEARS"

A 45 year old man with a 2 year forgotten left double-pigtail ureteric stent was referred to our hospital. Because of distal calcifications, the stent could not be removed by means of a cystoscopy. So, extracorporeal wave lithotripsies were performed and then the stent were easily removed in two fragments by cystoscopy and ureteroscopy.

KEY WORDS: Ureteral stent. Calcification. Extracorporeal wave lithotripsy.

$\mathrm{E}$ uso de catéteres ureterales tipo doble $\mathrm{J}$ es de gran utilidad en el tratamiento de la obstrucción ureteral. Sin embargo, ha de asumirse que la colocación de este tipo de catéteres no está exenta de problemas ya que hay pacientes que pueden presentar problemas de tolerancia y también desarrollar complicaciones como la fragmentación, la migración o la calcificación del catéter. No obstante, debido a la gran utilización de estos catéteres surge un nuevo problema que es el hecho de que se deje olvidado en el uréter. En este caso, no se es consciente del olvido hasta que se halla el catéter en un control rutinario o cuando empieza a causar síntomas debido a alguna complicación asociada. Una de las complicaciones más frecuentes es la calcificación e incluso la formación de litiasis en los extremos libres del catéter, tal como ocurrió en el caso clínico que se presenta. En esta situación, pensamos que la realización de litotricia extracorpórea con el fin de fragmentar la litiasis favorece extraordinariamente la extracción ulterior del catéter. 


\section{CASO CLÍNICO}

Varón de 45 años, alérgico al contraste yodado y con antecedentes de expulsión de litiasis sin complicaciones. Dos años antes, durante un viaje por Alemania presentó un cuadro compatible con cólico nefrítico izquierdo por el que fue atendido en un hospital. Allí fue sedado y sometido a manipulación por vía uretral que no recuerda completamente. Fue dado de alta y al encontrarse bien no consultó a su regreso a España. Dos años más tarde presentó escozor y polaquiuria. Consultó a un urólogo que encontró un catéter ureteral tipo doble $J$ calcificado en ambos extremos. Se intentó una extracción del mismo vía uretral no siendo posible debido a que la calcificación del extremo proximal estaba adherida a la vía urinaria. El paciente fue remitido a nuestro centro.

La Figura 1 muestra el catéter no fragmentado con sendas calcificaciones en ambos extremos. Con el fin de facilitar la extracción se realizó una sesión de litotricia mediante Lithostar Ultra ${ }^{\circledR}$ con 3.000 disparos sobre la calcificación proximal y

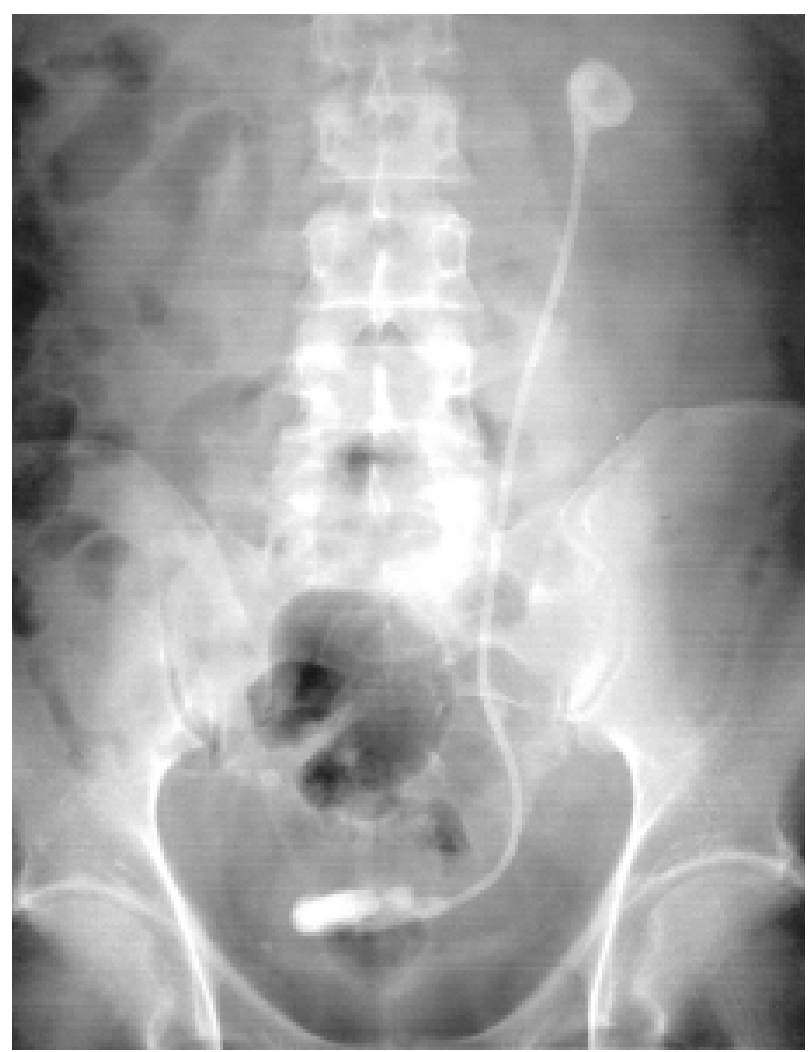

FIGURA 1. Radiografia simple renoureteral que muestra el catéter doble $J$ con ambos extremos calcificados.
4.000 sobre la vesical y a las 48 horas otra nueva sesión con 4.500 disparos sobre la calcificación proximal y 4.000 sobre la vesical. El resultado fue la desaparición de ambas calcificaciones con la formación de una calle litiásica a nivel de uréter lumbar (Fig. 2).

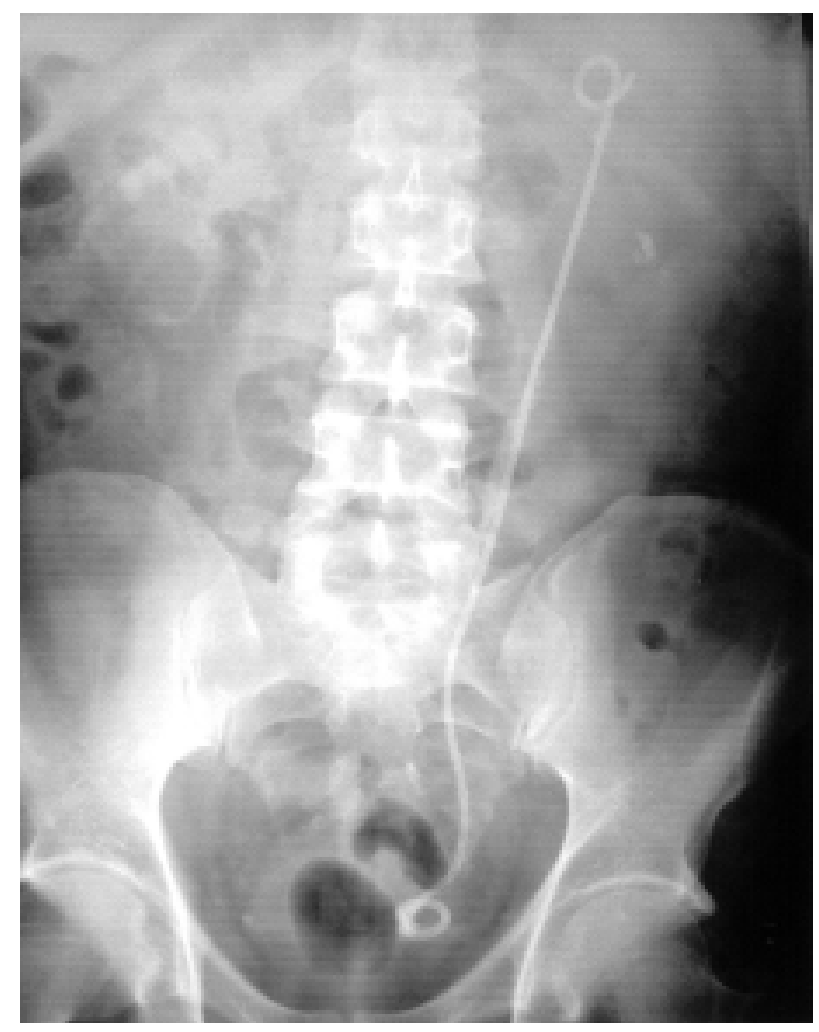

FIGURA 2. Radiografia simple renoureteral tras litotricia que muestra la desaparición de las calcificaciones de ambos extremos con una calle litiásica a nivel de uréter lumbar $L 4$.

Posteriormente, se procedió a retirar el catéter con una pinza flexible mediante cistoscopia, fragmentándose a nivel de la curvatura de uréter sacro, por lo que se requirió acabar de extraerlo mediante ureteroscopia (Fig. 3). No quedó ningún fragmento de catéter ni litiasis residual. El análisis de los fragmentos litiásicos reveló la existencia de 3 componentes: whewellita, carbonato apatita con una carbonatación muy intensa, y uno minoritario de brushita.

\section{DISCUSIÓN}

El uso de catéteres ureterales tipo doble $\mathrm{J}$ es de gran utilidad para la prevención o el tratamiento de la obstrucción ureteral independiente- 


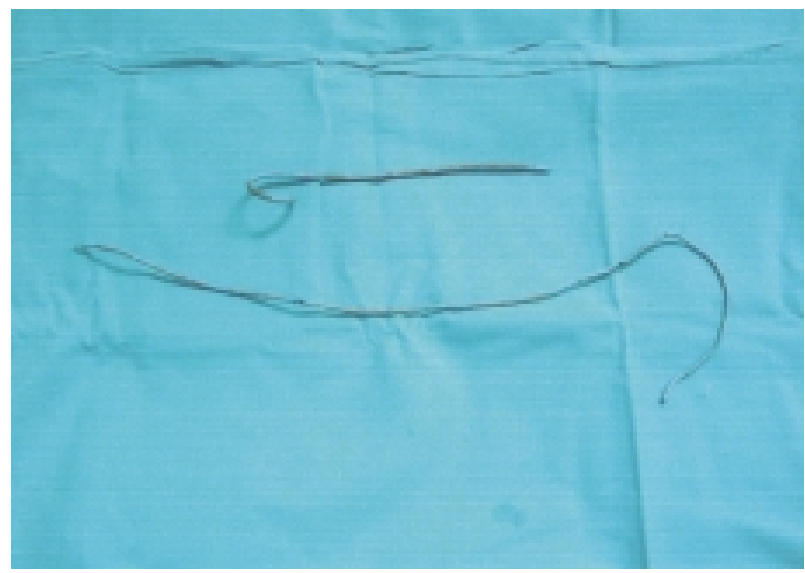

FIGURA 3. Catéter ureteral doble $J$ en dos fragmentos tras su extracción completa.

mente de la causa: litiasis, tumoral, estenosis, traumatismo, cirugía, radioterapia o embarazo. Sin embargo, a pesar de sus beneficios, podemos encontrar inconvenientes según la tolerancia del paciente y la aparición de posibles complicaciones. En cuanto a la tolerancia los síntomas más frecuentes que presentan los pacientes portadores de catéteres ureterales doble $\mathrm{J}$ son el dolor suprapúbico y en el flanco que se manifiesta sobre todo durante la micción y en menor medida caminando o en decúbito ${ }^{1}$. Esta aparición de dolor en el flanco durante la micción se atribuye al reflujo que provoca el catéter ${ }^{2-3}$. Asimismo, el estímulo del catéter en la mucosa vesical provoca la aparición de síntomas irritativos caracterizados por polaquiuria y urgencia. Por otro lado, en ocasiones aparecen complicaciones que requieren la retirada precoz del catéter: no desaparición de la hidronefrosis $(9,1 \%)$, empeoramiento de la hidronefrosis $(2,7 \%)$, aparición de hidronefrosis $(2,7 \%)$, migración del catéter $(8,2 \%)$ y fragmentación del mismo $(10 \%)^{4}$. Las complicaciones infecciosas precoces como la fiebre y la bacteriuria suelen aparecer en la primera semana tras la colocación del catéter.

No obstante, otros problemas que indirectamente pueden causar los catéteres ureterales doble $\mathrm{J}$ son los derivados del olvido del catéter. Son varios los casos publicados, siendo el más extremo un catéter olvidado durante 10 años ${ }^{5}$ que incluso perdió su revestimiento radiopaco por lo que no podía ser evidenciado mediante radiología simple. Las complicaciones más frecuentes del olvido de estos catéteres son la fragmentación, la calcificación y formación de cálculos y en casos extremos la incorporación del mismo a la pared ureteral $^{6}$. La fragmentación puede producirse incluso en catéteres que llevan colocados un período breve de tiempo ${ }^{4,7}$. En cualquier caso, para extraer el catéter se necesita una maniobra combinada de abordaje percutáneo para extraer el extremo superior y además una ureteroscopia o cistoscopia para extraer el inferior ${ }^{7-8}$. Sin embargo, en ocasiones debido a que la fragmentación es múltiple o a la adherencia del catéter a la mucosa ureteral ha de recurrirse a la cirugia abierta ${ }^{6,9}$.

La otra complicación de un catéter olvidado es la calcificación del mismo y muy frecuentemente la aparición de litiasis sobre todo en su extremo proximal piélico y en el distal vesical. Este fenómeno es más frecuente en aquellos pacientes con predisposición a desarrollar cálculos. Esto puede suponer un fracaso del tratamiento, concretamente si el catéter se había colocado para resolver litiasis de menor tamaño. La formación de litiasis en los extremos del catéter dificulta su extracción ya que suele presentar un tamaño superior al de la unión pieloureteral o al del meato ureteral y además la litiasis suele estar adherida en la mucosa urotelial. De este modo, para extraer el catéter, independientemente de la vía de abordaje, conviene deshacer previamente estas litiasis. Hay casos en los que se va directamente a la cirugía percutánea ${ }^{9} \mathrm{y}$ otros en los que previamente se realiza una litotricia electrohidráulica endovesical ${ }^{8}$. Sin embargo, tal como muestran otros autores ${ }^{8,10}$ y el caso clínico presentado, lo más sencillo es realizar previamente litotricia extracorpórea sobre las litiasis de los extremos hasta conseguir su fragmentación. Si es necesario, se realiza más de una sesión hasta obtener una limpieza de los extremos del catéter. Una vez que se ha conseguido se procede a la retirada del mismo intentando hacerlo de la forma menos agresiva. Primero mediante cistoscopia y extracción con pinza. Si no se consigue o se fragmenta el catéter se procede a extraerlo mediante ureteroscopia. Como última opción, si no se consigue o queda un extremo perdido en pelvis se extrae mediante cirugía percutánea. En cualquier caso, independientemente del abordaje que se acabe realizando, la maniobra extractora se ve favorecida por el tratamiento previo de los extremos calcificados mediante litotricia extracorpórea. 


\section{REFERENCIAS}

1. IRANI J, SIQUIER C, PIRÈS O et al.: Symptom characteristics and the development of tolerance with time in patients with indwelling double-pigtail ureteric stents. BJU International 1999; 84: 276-279.

2. POLLARD SG, MACFARLANE R.: Symptoms arising form double J ureteral stents. J Urol 1988; 139: 3738.

3. MOSLI HA, FARSI HM, AL-ZIMAITY MF et al.: Vesicoureteral reflux in patients with double pigtail stents. J Urol 1991; 146: 966-969.

4. RINGEL A, RICHTER S, SHALEV M et al.: Late complications of ureteral stents. Eur Urol 2000; 38: 41-44.

5. McKIERNAN JM, KATZ AE, GIKYBIFF et al.: A longforgotten ureteral stent. Urology 1997; 49: 622-623.

6. IVIL KD, SURESH G.: Incorporation of a forgotten stent into the ureteral wall. J Urol 2001; 165: 19911992.

7. ZISMAN A, SIEGEL YI, SIEGMANN A et al.: Spontaneous ureteral stent fragmentation. J Urol 1995: 153: 718-721.
8. MONGA M, KLEIN E, CASTAÑEDA-ZUÑIGA WR et al.: The forgotten indwelling ureteral stent: a urological dilemma. J Urol 1995; 153: 1817-1819.

9. REMBRINK K, GOEPEL M, MEYER-SCHWICKERATH M.: The forgotten double J stent. Urol Int 1992; 49: 119-120.

10. SOMERS WJ.: Management of forgotten or retained indwelling ureteral stents. Urology 1996; 47: 431435.

Dr. F. Millán Rodríguez

Servicio de Urología

Fundación Puigvert

C/ Cartagena, 340-350

08025 Barcelona

(Trabajo recibido el 31 octubre de 2001) 